University of Wollongong

Research Online

Faculty of Informatics - Papers (Archive)

Faculty of Engineering and Information

Sciences

$1-1-2008$

\title{
Application of Quasi-orthogonal space-time-frequency codes in MB-OFDM UWB
}

Alfred Mertins

University of Luebeck, Germany, mertins@uow.edu.au

Le Chung Tran

University of Wollongong, Ictran@uow.edu.au

Follow this and additional works at: https://ro.uow.edu.au/infopapers

Part of the Physical Sciences and Mathematics Commons

\section{Recommended Citation}

Mertins, Alfred and Tran, Le Chung: Application of Quasi-orthogonal space-time-frequency codes in MBOFDM UWB 2008, 73-76.

https://ro.uow.edu.au/infopapers/788

Research Online is the open access institutional repository for the University of Wollongong. For further information contact the UOW Library: research-pubs@uow.edu.au 


\title{
Application of Quasi-orthogonal space-time-frequency codes in MB-OFDM UWB
}

\author{
Abstract \\ This paper examines the application of Quasi-Orthogonal Space-Time-Frequency Codes (QOSTFCs) to \\ advance either data rate or error performance in recently proposed Space-Time-Frequency Coded \\ Multiband OFDM Ultra-Wideband (STFC MB-OFDM UWB) communications systems. It is shown that \\ QOSTFCs can provide significantly better error performance, compared to the conventional MB-OFDM \\ UWB (without STFCs) and to the Orthogonal STFCs (OSTFCs) of the same order, at the same data rate, \\ without increasing the total transmission power. In other words, QOSTFCs can provide higher data rates \\ with the same error performance, compared to OSTFCs.

\section{Keywords} \\ space, orthogonal, quasi, application, mb, codes, frequency, uwb, time, ofdm \\ Disciplines \\ Physical Sciences and Mathematics

\section{Publication Details} \\ Tran, L. C. \& Mertins, A. (2008). Application of Quasi-orthogonal space-time-frequency codes in MB-OFDM \\ UWB. Proc. IEEE International Conference on Ultra-Wideband (ICUWB 2008) (invited paper) (pp. 73-76). \\ Hannover, Germany: Institute of Electrical and Electronics Engineers ( IEEE ).
}




\title{
Application of Quasi-Orthogonal Space-Time-Frequency Codes in MB-OFDM UWB
}

\author{
L. C. Tran and A. Mertins \\ University of Luebeck, Germany \\ Email: \{tran,mertins\}@isip.uni-luebeck.de
}

\begin{abstract}
This paper examines the application of QuasiOrthogonal Space-Time-Frequency Codes (QOSTFCs) to advance either data rate or error performance in recently proposed Space-Time-Frequency Coded Multiband OFDM UltraWideband (STFC MB-OFDM UWB) communications systems. It is shown that QOSTFCs can provide significantly better error performance, compared to the conventional MB-OFDM UWB (without STFCs) and to the Orthogonal STFCs (OSTFCs) of the same order, at the same data rate, without increasing the total transmission power. In other words, QOSTFCs can provide higher data rates with the same error performance, compared to OSTFCs.
\end{abstract}

\section{INTRODUCTION}

Combination of the emerging technologies Multiband OFDM Ultra-Wideband (MB-OFDM UWB), Multiple Input Multiple Output (MIMO), and Space-Time Codes (STCs) may provide a significant improvement in the maximum achievable wireless communications range, bit error performance, system capacity, and data rate. This combination has been mentioned in the literature. In particular, the combination of MB-OFDM UWB and Space-Time Block Codes (STBCs) has been mentioned in [1] for only 2 transmit antennas (the Alamouti code [2]). In [3], the authors proposed a general framework to analyze the performance of MB-OFDM MIMO UWB systems regardless of specific coding schemes in case of Nakagami frequency-selective fading channels. In [4], [5], we proposed the Space-Time-Frequency Coded MB-OFDM UWB (STFC MB-OFDM UWB) system for any number of transmit (Tx) and receive (Rx) antennas (see Fig. 1). We modified Tarokh's proof [6] to quantify the diversity and coding gains of the proposed STFC MB-OFDM UWB system in the log-normal distribution case. We also derived the design criteria and maximum likelihood (ML) decoding expressions for STFCs in MB-OFDM UWB.

One disadvantage of STFCs, which are constructed based directly on complex Orthogonal STBCs (OSTBCs) as mentioned in [4], is the reduced code rate when the number of Tx antennas increases. It is well known that OSTBCs for more than $2 \mathrm{Tx}$ antennas cannot provide the full rate. To increase the code rate, in [7], the author proposed QuasiOrthogonal STBCs (QOSTBCs) for 4 and 8 Tx antennas, providing higher data rates than the conventional OSTBCs of the same orders, while they still can provide a large (but not full) diversity. Equivalently, QOSTBCs can provide better error performance, compared to OSTBCs of the same order, at the same data rate [7]. The idea of QOSTBCs [7] can definitely be further extended to apply to STFCs in MB-OFDM UWB.

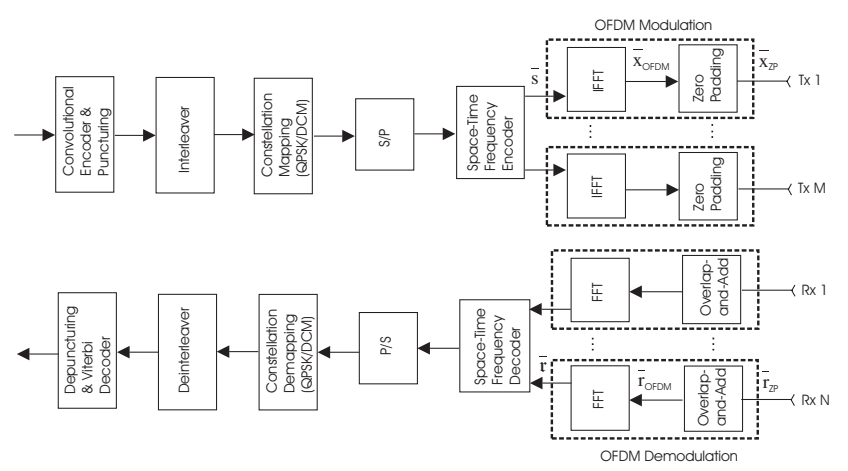

Fig. 1. Structural diagram of the proposed STFC MB-OFDM UWB systems.

Therefore, in this paper, we consider the application of QuasiOrthogonal STFCs (QOSTFCs), which have higher code rates than orthogonal STFCs (OSTFCs) of the same order, in the proposed STFC MB-OFDM UWB system. It will be shown that, although only partial diversity can be achieved, QOSTFCs may still provide better error performance over OSTFCs of the same order, without any increase of the total transmission power.

\section{QUASI-ORTHOGONAL STFCS}

For the code rate of a STFC [4] being defined as the ratio of the number of transmitted MB-OFDM symbols and the number of time slots required to transmit the whole block of the code, it is known that the Alamouti code [2] can provide a full rate for two Tx antennas, while higher-order codes ${ }^{1}$ for more than two Tx antennas cannot provide the full rate (except when some special techniques such as [8], [9] are used to increase the code rate). However, they may still provide a higher diversity order than the Alamouti STFC. As a result, the higher-order codes may provide better error performance without any increase of the total transmission power. Higher order codes also provide potentially higher capacity for the wireless system. We note that, while high order codes (order 8 or greater) do not bring about a significant increase of the system capacity in the case $N=1 \mathrm{Rx}$ antenna (see Fig. 2.2. in [10]), they may significantly increase the system capacity in the case $N \geq 2 \mathrm{Rx}$ antennas (see Fig. 2.4. in [10] for instance). Therefore, the implementation of higher-order STFCs for multiple Tx/Rx antennas in STFC MB-OFDM UWB communications is still of our interest.

\footnotetext{
${ }^{1}$ The order of a STFC in this paper refers to the number of rows in that code matrix. In other words, it refers to the number of Tx antennas required to transmit the whole code block.
} 
A question that could be raised is: what is the possible maximum order of STFCs which may be practically applied to MB-OFDM UWB systems? It is well known that Tx antennas should be separated from one another by at least $\lambda / 2$, where $\lambda$ is the UWB wavelength, in order to avoid the spatial correlation between the Tx antennas. For the UWB frequency range $3.1-10.6 \mathrm{GHz}$, this minimum distance is in the range of 14.2-48.4 $\mathrm{mm}$. Let us consider the implementation of order8 STFCs ( $8 \mathrm{Tx}$ antennas are required). The length of UWB devices locating $8 \mathrm{Tx}$ antennas should be about $7 \lambda / 2$, i.e. in the range $9.9-33.9 \mathrm{~cm}$. This length is the typical length of wireless devices, such as wireless access points or routers. For more than $8 \mathrm{Tx}$ antennas, the physical size of UWB devices might be too large and thus impractical. Therefore, the application of up to $8 \mathrm{Tx}$ antennas can be feasible in STFC MB-OFDM UWB.

As mentioned previously in the introduction, QOSTFCs can be used to improve either the data rate or the error performance of MB-OFDM UWB systems. In particular, for 4 Tx antennas, the following full rate QOSTFC $S_{4 b}$, which is constructed in the similar way as the QOSTBC proposed in [7], can be applied

$$
\mathbf{S}_{4 \mathbf{b}}=\left[\begin{array}{rrrr}
\overline{\mathbf{s}}_{1} & \overline{\mathbf{s}}_{2} & \overline{\mathbf{s}}_{3} & \overline{\mathbf{s}}_{4} \\
-\overline{\mathbf{s}}_{2}^{*} & \overline{\mathbf{s}}_{1}^{*} & -\overline{\mathbf{s}}_{4}^{*} & \overline{\mathbf{s}}_{3}^{*} \\
-\overline{\mathbf{s}}_{3}^{*} & -\overline{\mathbf{s}}_{4}^{*} & \overline{\mathbf{s}}_{1}^{*} & \overline{\mathbf{s}}_{2}^{*} \\
\overline{\mathbf{s}}_{4} & -\overline{\mathbf{s}}_{3} & -\overline{\mathbf{s}}_{2} & \overline{\mathbf{s}}_{1}
\end{array}\right],
$$

where $\overline{\mathbf{s}}_{i}$ denotes a length- $N_{f f t}$ column vector, while $N_{f f t}$ is the FFT/IFFT size. This QOSTFC provides a higher code rate with the penalty of loosing half diversity order, compared to the rate-3/4 OSTFC $S_{4 a}$, which is constructed based on the code proposed in [11] for conventional wireless STBC MIMO systems

$$
\mathbf{S}_{4 \mathbf{a}}=\left[\begin{array}{rrrr}
\overline{\mathbf{s}}_{1} & \overline{\mathbf{s}}_{2} & \overline{\mathbf{s}}_{3} & 0 \\
-\overline{\mathbf{s}}_{2}^{*} & \overline{\mathbf{s}}_{1}^{*} & 0 & \overline{\mathbf{s}}_{3} \\
-\overline{\mathbf{s}}_{3}^{*} & 0 & \overline{\mathbf{s}}_{1}^{*} & -\overline{\mathbf{s}}_{2} \\
0 & -\overline{\mathbf{s}}_{3}^{*} & \overline{\mathbf{s}}_{2}^{*} & \overline{\mathbf{s}}_{1}
\end{array}\right] .
$$

For 8 Tx antennas, the following rate-3/4 QOSTFC $S_{8 b}$ (constructed in the similar way as the QOSTBC proposed in [7]) can be used

$$
\mathbf{S}_{\mathbf{8 b}}=\left[\begin{array}{rrrrrrrr}
\overline{\mathbf{s}}_{1} & \overline{\mathbf{s}}_{2} & \overline{\mathbf{s}}_{3} & 0 & \overline{\mathbf{s}}_{4} & \overline{\mathbf{s}}_{5} & \overline{\mathbf{s}}_{6} & 0 \\
-\overline{\mathbf{s}}_{2}^{*} & \overline{\mathbf{s}}_{1}^{*} & 0 & -\overline{\mathbf{s}}_{3} & \overline{\mathbf{s}}_{5}^{*} & -\overline{\mathbf{s}}_{4}^{*} & 0 & \overline{\mathbf{s}}_{6} \\
\overline{\mathbf{s}}_{3}^{*} & 0 & -\overline{\mathbf{s}}_{1}^{*} & -\overline{\mathbf{s}}_{2} & -\overline{\mathbf{s}}_{6}^{*} & 0 & \overline{\mathbf{s}}_{4}^{*} & \overline{\mathbf{s}}_{5} \\
0 & -\overline{\mathbf{s}}_{3}^{*} & \overline{\mathbf{s}}_{2}^{*} & -\overline{\mathbf{s}}_{1} & 0 & \overline{\mathbf{s}}_{6}^{*} & -\overline{\mathbf{s}}_{5}^{*} & \overline{\mathbf{s}}_{4} \\
-\overline{\mathbf{s}}_{4} & -\overline{\mathbf{s}}_{5} & -\overline{\mathbf{s}}_{6} & 0 & \overline{\mathbf{s}}_{1} & \overline{\mathbf{s}}_{2} & \overline{\mathbf{s}}_{3} & 0 \\
-\overline{\mathbf{s}}_{5}^{*} & \overline{\mathbf{s}}_{4}^{*} & 0 & \overline{\mathbf{s}}_{6} & -\overline{\mathbf{s}}_{2}^{*} & \overline{\mathbf{s}}_{1}^{*} & 0 & \overline{\mathbf{s}}_{3} \\
\overline{\mathbf{s}}_{6}^{*} & 0 & -\overline{\mathbf{s}}_{4}^{*} & \overline{\mathbf{s}}_{5} & \overline{\mathbf{s}}_{3}^{*} & 0 & -\overline{\mathbf{s}}_{1}^{*} & \overline{\mathbf{s}}_{2} \\
0 & \overline{\mathbf{s}}_{6}^{*} & -\overline{\mathbf{s}}_{5}^{*} & -\overline{\mathbf{s}}_{4} & 0 & \overline{\mathbf{s}}_{3}^{*} & -\overline{\mathbf{s}}_{2}^{*} & -\overline{\mathbf{s}}_{1}
\end{array}\right]
$$

This QOSTFC provides a higher code rate with the penalty of loosing a portion of maximum diversity order, compared to the following rate-1/2 OSTFC $S_{8 a}$, which is constructed based on the code proposed in [11] for conventional wireless STBC MIMO systems

$$
\mathbf{S}_{\mathbf{8 a}}=\left[\begin{array}{rrrrrrrr}
\overline{\mathbf{s}}_{1} & -\overline{\mathbf{s}}_{2}^{*} & -\overline{\mathbf{s}}_{3}^{*} & 0 & -\overline{\mathbf{s}}_{4}^{*} & 0 & 0 & 0 \\
\overline{\mathbf{s}}_{2} & \overline{\mathbf{s}}_{1}^{*} & 0 & \overline{\mathbf{s}}_{3}^{*} & 0 & \overline{\mathbf{s}}_{4}^{*} & 0 & 0 \\
\overline{\mathbf{s}}_{3} & 0 & \overline{\mathbf{s}}_{1}^{*} & -\overline{\mathbf{s}}_{2}^{*} & 0 & 0 & \overline{\mathbf{s}}_{4}^{*} & 0 \\
0 & -\overline{\mathbf{s}}_{3} & \overline{\mathbf{s}}_{2} & \overline{\mathbf{s}}_{1} & 0 & 0 & 0 & -\overline{\mathbf{s}}_{4}^{*} \\
\overline{\mathbf{s}}_{4} & 0 & 0 & 0 & \overline{\mathbf{s}}_{1}^{*} & -\overline{\mathbf{s}}_{2}^{*} & -\overline{\mathbf{s}}_{3}^{*} & 0 \\
0 & -\overline{\mathbf{s}}_{4} & 0 & 0 & \overline{\mathbf{s}}_{2} & \overline{\mathbf{s}}_{1} & 0 & \overline{\mathbf{s}}_{3}^{*} \\
0 & 0 & -\overline{\mathbf{s}}_{4} & 0 & \overline{\mathbf{s}}_{3} & 0 & \overline{\mathbf{s}}_{1} & -\overline{\mathbf{s}}_{2}^{*} \\
0 & 0 & 0 & \overline{\mathbf{s}}_{4} & 0 & -\overline{\mathbf{s}}_{3} & \overline{\mathbf{s}}_{2} & \overline{\mathbf{s}}_{1}^{*}
\end{array}\right]
$$

It can be realized that the columns $\nu_{i}$, for $i=1, \ldots 8$, of

\begin{tabular}{|c|c|}
\hline \multirow{3}{*}{$\frac{\text { Symbols }}{\left(\overline{\mathbf{s}}_{1}, \overline{\mathbf{s}}_{4}\right)}$} & Decoding Metric \\
\hline & $\arg \min _{\overline{\mathbf{s}}_{1}, \overline{\mathbf{s}}_{4} \in \mathcal{C}^{N} D} \|\left(\sum_{m=1}^{4} \sum_{n=1}^{N}\left|\overline{\mathfrak{h}}_{m, n}\right| \cdot{ }^{\wedge} 2\right) \bullet$ \\
\hline & $\begin{array}{l}\left(\left|\overline{\mathbf{s}}_{1}\right| \cdot \wedge^{\wedge}+\left|\overline{\mathbf{s}}_{4}\right| \cdot{ }^{\wedge} 2\right)+2 \operatorname{Real}\left[\sum_{n=1}^{N}\left(-\overline{\mathfrak{h}}_{1, n} \bullet \overline{\mathfrak{r}}_{1, n}^{*}-\right.\right. \\
\left.\left.\overline{\mathfrak{h}}_{2, n}^{*} \bullet \overline{\mathfrak{r}}_{2, n}-\overline{\mathfrak{h}}_{3, n}^{*} \bullet \overline{\mathfrak{r}}_{3, n}-\overline{\mathfrak{h}}_{4, n} \bullet \overline{\mathfrak{r}}_{4, n}^{*}\right) \bullet \overline{\mathbf{s}}_{1}\right]+ \\
2 \operatorname{Real}\left[\sum_{n=1}^{N}\left(-\overline{\mathfrak{h}}_{4, n} \bullet \overline{\mathfrak{r}}_{1, n}^{*}+\overline{\mathfrak{h}}_{3, n}^{*} \bullet \overline{\mathfrak{r}}_{2, n}+\overline{\mathfrak{h}}_{2, n}^{*} \bullet \overline{\mathfrak{r}}_{3, n}-\right.\right. \\
\left.\left.\overline{\mathfrak{h}}_{1, n} \bullet \overline{\mathfrak{r}}_{4, n}^{*}\right) \bullet \overline{\mathbf{s}}_{4}\right]+2 \operatorname{Real}\left[\sum_{n=1}^{N}\left(\overline{\mathfrak{h}}_{1, n} \bullet \overline{\mathfrak{h}}_{4, n}^{*}-\right.\right. \\
\left.\left.\overline{\mathfrak{h}}_{2, n}^{*} \bullet \overline{\mathfrak{h}}_{3, n}-\overline{\mathfrak{h}}_{2, n} \bullet \overline{\mathfrak{h}}_{3, n}^{*}+\overline{\mathfrak{h}}_{1, n}^{*} \bullet \overline{\mathfrak{h}}_{4, n}\right) \bullet \overline{\mathbf{s}}_{1} \bullet \overline{\mathbf{s}}_{4}^{*}\right] \|_{F}^{2}\end{array}$ \\
\hline$\left(\overline{\mathbf{s}}_{2}, \overline{\mathbf{s}}_{3}\right)$ & 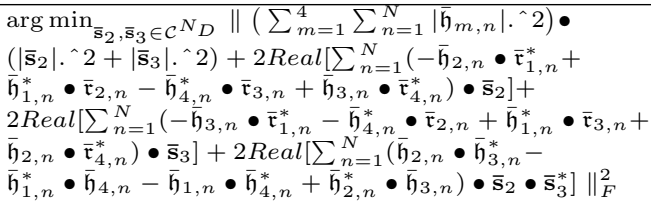 \\
\hline
\end{tabular}
$S_{8 b}$ are orthogonal, except for the pairs $\left(\nu_{1}, \nu_{5}\right),\left(\nu_{2}, \nu_{6}\right)$ and
TABLE I

Decoding Metrics For $\mathbf{S}_{\mathbf{4 b}}$ With PSK or QAM Modulations.

$\left(\nu_{3}, \nu_{7}\right)$. Clearly, the orthogonality of $S_{8 b}$ (thus the diversity) is partially released to achieve the higher rate.

We now derive the ML decoding metrics for the above QOSTFCs in a MIMO system with $N \mathrm{Rx}$ antennas, using a PSK or QAM modulation scheme. Let us consider the two codes $\mathbf{S}_{8 a}$ and $\mathbf{S}_{8 b}$ as examples. Because $\mathbf{S}_{8 a}$ is completely orthogonal and $\mathbf{S}_{8 b}$ is partially orthogonal, each MB-OFDM symbol can be decoded separately for the code $\mathbf{S}_{8 a}$, while a pair of MB-OFDM symbols must be decoded at a time for the code $\mathbf{S}_{8 b}$. The decoding metrics of MB-OFDM symbols in the two codes can be easily found based on the decoding metrics of the respective OSTBC (see, for example, [10, Section 3.3]) and QOSTBC [7, Section III]. The decoding process is further simplified by the fact that, instead of having to decode jointly the whole $N_{D}$ data points corresponding to the $N_{D}$ data subcarriers $\left(N_{D}=100\right.$ according to [12]) within a MB-OFDM symbol $\overline{\mathbf{s}}_{t, m}$, each data point among these $N_{D}$ data subcarriers (a pair of data points for QOSTFCs) can be decoded separately. Thus the decoding process is relatively simple. The decoding metrics of MB-OFDM symbols for $\mathbf{S}_{4 b}$ and $\mathbf{S}_{8 b}$ are presented in Tables I and II respectively, while those for $\mathbf{S}_{4 a}$ and $\mathbf{S}_{8 a}$ can be found in [13, Table II] and [14] respectively which are not repeated in this paper. In these tables, $\overline{\mathfrak{h}}_{m, n}$ is the column vector obtained by applying the FFT to the respective channel coefficient vector $\overline{\mathbf{h}}_{m, n}$, i.e. $\overline{\mathfrak{h}}_{m, n}=F F T\left\{\overline{\mathbf{h}}_{m, n}\right\}$, while $\overline{\mathfrak{r}}_{m, n}$ is the column vector of received signals at the output of the FFT blocks in Fig. 1. Once again, we refer readers to $[4$, Section III A] for more detail about how these vectors are formed.

\section{Practical Issues of the Application of QOSTFCS}

In realistic MB-OFDM UWB systems [12], multiple symbols are transmitted within a PSDU (PLCP Service Data Units) that is headed by a PLCP (Physical Layer Convergence Protocol) preamble consisting of 24 OFDM symbols (12 symbols in the case of burst mode) for packet and frame synchronization and 6 OFDM symbols for channel estimation. Also, different types of TFCs (Time Frequency Codes) are used for transmission. Let us consider the first band group of WiMedia Alliance's MB-OFDM UWB systems (the radio frequency is in the range of $3.168-4.752 \mathrm{GHz}$ ) as an example. There are 7 TFCs (the RF patterns) defined for this band 
TABLE II

DeCoding METRICS FOR $\mathbf{S}_{\mathbf{8} \mathbf{b}}$ WiTh PSK OR QAM Modulations.

\begin{tabular}{|c|c|}
\hline Symbols & Decoding Metric \\
\hline$\left(\overline{\mathbf{s}}_{1}, \overline{\mathbf{s}}_{4}\right)$ & 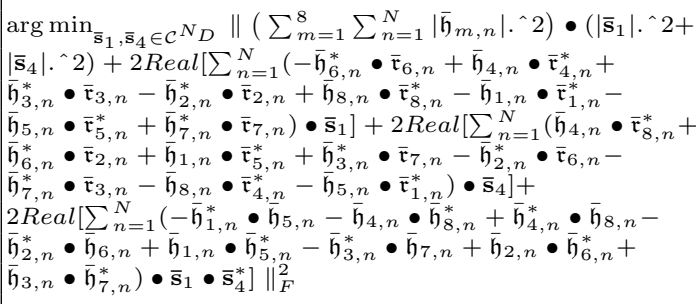 \\
\hline$\left(\overline{\mathbf{s}}_{2}, \overline{\mathbf{s}}_{5}\right)$ & 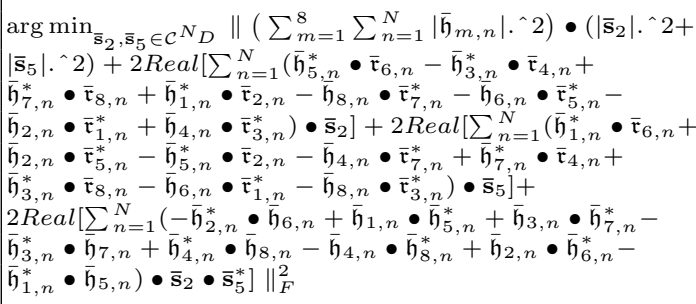 \\
\hline$\left(\overline{\mathbf{s}}_{3}, \overline{\mathbf{s}}_{6}\right)$ & 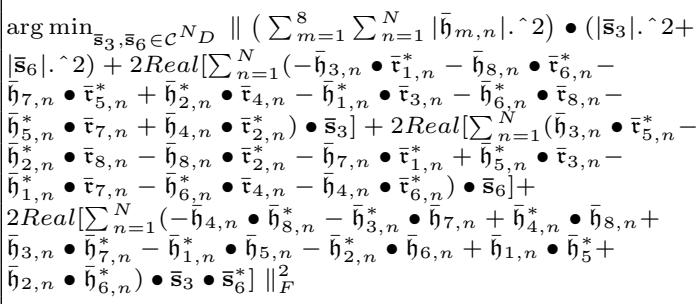 \\
\hline
\end{tabular}

group [12, Table 7-2], divided into two types, namely TimeFrequency Interleaving (TFI), where MB-OFDM symbols are interleaved over three sub-bands within this band group, and Fixed Frequency Interleaving (FFI), where MB-OFDM symbols are transmitted on a single sub-band. The TFCs 1,2 , 3 and 4 are belong to TFI while the TFCs 5, 6, 7 are belong to FFI. Support for both TFI and FFI is mandatory. In the case of TFI, among 6 OFDM symbols used for channel estimation, a pair of OFDM symbols is used for channel estimation in each of the three sub-bands respectively, while in the case of FFI, all of these six symbols can be used for channel estimation in one sub-band. The estimated channel vectors shall be then used to decode all symbols transmitted within the PSDU though channel vectors may in fact vary (more or less) in time during the transmission duration of the PSDU.

We will now consider the application of QOSTFCs in realistic MB-OFDM UWB systems with multiple Tx/Rx antennas. In particular, for the MB-OFDM UWB system with a 4IMO (4 Tx antennas) configuration, the QOSTFC $\mathbf{S}_{4 b}$ can be used with the four Tx antennas transmitting the following symbol sequences respectively

$$
\begin{aligned}
& {\left[\overline{\mathbf{s}}_{1} \overline{\mathbf{s}}_{2} \overline{\mathbf{s}}_{3} \overline{\mathbf{s}}_{4} \ldots \overline{\mathbf{s}}_{M-3} \overline{\mathbf{s}}_{M-2} \overline{\mathbf{s}}_{M-1} \overline{\mathbf{s}}_{M}\right]} \\
& {\left[-\overline{\mathbf{s}}_{2}^{*} \overline{\mathbf{s}}_{1}^{*}-\overline{\mathbf{s}}_{4}^{*} \overline{\mathbf{s}}_{3}^{*} \ldots-\overline{\mathbf{s}}_{M-2}^{*} \overline{\mathbf{s}}_{M-3}^{*}-\overline{\mathbf{s}}_{M}^{*} \overline{\mathbf{s}}_{M-1}^{*}\right]} \\
& {\left[-\overline{\mathbf{s}}_{3}^{*}-\overline{\mathbf{s}}_{4}^{*} \overline{\mathbf{s}}_{1}^{*} \overline{\mathbf{s}}_{2}^{*} \ldots-\overline{\mathbf{s}}_{M-1}^{*}-\overline{\mathbf{s}}_{M}^{*} \overline{\mathbf{s}}_{M-3}^{*} \overline{\mathbf{s}}_{M-2}^{*}\right]} \\
& {\left[\overline{\mathbf{s}}_{4}-\overline{\mathbf{s}}_{3}-\overline{\mathbf{s}}_{2} \overline{\mathbf{s}}_{1} \ldots \overline{\mathbf{s}}_{M}-\overline{\mathbf{s}}_{M-1}-\overline{\mathbf{s}}_{M-2} \overline{\mathbf{s}}_{M-3}\right] \text {. }}
\end{aligned}
$$

The MB-OFDM symbols in each of the above formulas are transmitted within the payload field of PSDUs. It is noted that, by the above formulas, we are assuming that there are total $M$ MB-OFDM symbols (corresponding to $M / 4$ code blocks $\mathbf{S}_{4 b}$ ) transmitted within the payload field of each PSDU. The transmission procedure in an 8IMO MB-OFDM UWB system with the QOSTFC $\mathbf{S}_{8 b}$ can be similarly deduced without any difficulty.

The ML decoding method mentioned in Section II requires that the same channel vectors must be used during at least four time slots (MB-OFDM symbol time slots) in order for each code block $\mathbf{S}_{4 b}$ to be correctly decoded. Clearly, if one of the first four TFCs, where consecutive MB-OFDM symbols are transmitted on different frequency sub-bands from a certain Tx antenna, is used, the channel vector(s) from this Tx antenna to the Rx antenna(s) cannot be the same for consecutive MBOFDM symbols. However, this will not be the case if one of the last three TFCs, i.e. TFCs 5, 6 or 7, where all MB-OFDM symbols are transmitted on a single sub-band, is utilized.

Therefore, QOSTFCs can be readily applied for STFC MB-OFDM UWB systems whenever FFI is used. In our aforementioned example, the MB-OFDM symbols in Eq. (5) (similarly for (6), (7) and (8)) shall be transmitted within a PSDU on the same sub-band, using one of the three TFCs 5, 6 and 7. If the receiver is equipped with only one Rx antenna, all six OFDM symbols in the preamble associated with this PSDU might be used to estimate the channel vector between the Tx antenna of interest and the Rx antenna. However, if the receiver is equipped with three Rx antennas, for instance, each pair of these six OFDM symbols can be used for channel estimation between the Tx antenna of interest and one of three $\mathrm{Rx}$ antennas respectively.

\section{Simulation Results and Discussions}

We ran several simulations for the conventional MB-OFDM without STFCs, and for $\mathbf{S}_{4 a}, \mathbf{S}_{4 b}, \mathbf{S}_{8 a}$, and $\mathbf{S}_{8 b}$, at the bit rate $480 \mathrm{Mbps}$ for illustration. Each run of simulations was carried out with 1800 MB-OFDM symbols. One hundred channel realizations of each of the four channel models CM1, CM2, CM3 and CM4 [15]) were considered for the transmission of each MB-OFDM symbol. To fairly compare the error performance of MB-OFDM systems with and without STFCs, the following two constraints are guaranteed

Data rate constraint: A suitable modulation scheme is selected for each MB-OFDM system in order to achieve the same data rate (for instance, we consider the basic MB-OFDM system of data rate $320 \mathrm{Mbps}$ with the convolutional encoder's rate of 1/2 (see Fig. 1), but selecting 8PSK (instead of QPSK or DCM - Dual Carrier Modulation) for the conventional MBOFDM, 16QAM for $\mathbf{S}_{4 a}$, 8PSK for $\mathbf{S}_{4 b}, 64 \mathrm{QAM}$ for $\mathbf{S}_{8 a}$, and 16QAM for $\mathbf{S}_{8 b}$ to have the same data rate of $480 \mathrm{Mbps}$ ).

Power constraint: We assume that all of the 8PSK, 16QAM and 64QAM signal constellations originally have an unit average power. This original average power is now scaled down by a factor of 1 for the uncoded MB-OFDM, 1/3 for $\mathbf{S}_{4 a}, 1 / 4$ for $\mathbf{S}_{4 b}, 1 / 4$ for $\mathbf{S}_{8 a}$, and $1 / 6$ for $\mathbf{S}_{8 b}$. Thereby, the total transmission power from all $\mathrm{Tx}$ antennas at a certain time is the same for all cases.

From Figs. 2(a) and 2(b), we can have the two following realizations. First, although only partial diversity can be 


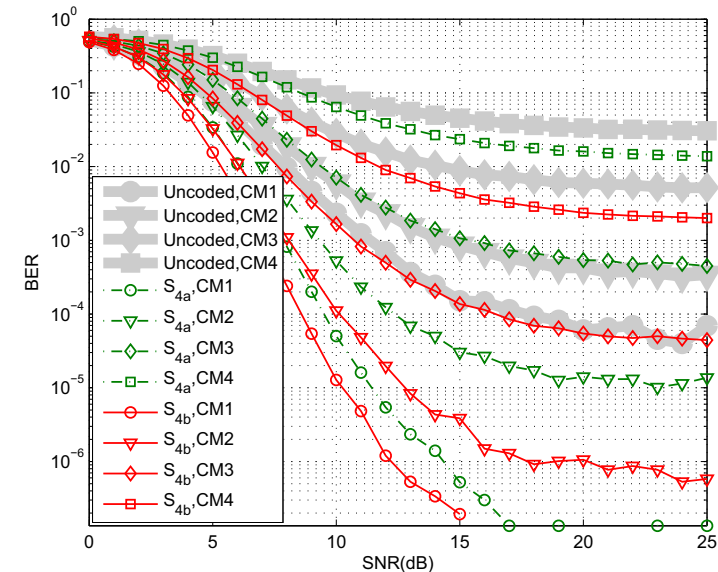

(a) Uncoded: 8PSK, $S_{4 a}$ : 16QAM, $S_{4 b}$ : 8PSK

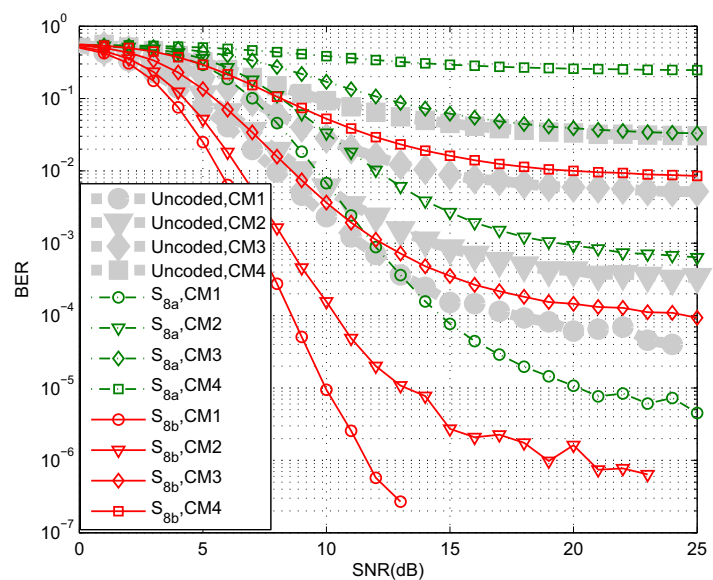

(b) Uncoded: 8PSK, $S_{8 a}:$ 64QAM, $S_{8 b}:$ 16QAM

Fig. 2. (a) $S_{4 b}$ vs. $S_{4 a}$, and (b) $S_{8 b}$ vs. $S_{8 a}$, with $2 \mathrm{Rx}$ antennas at the bit rate $480 \mathrm{Mbps}$.

achieved, the QOSTFCs can still provide significantly better error performance (at least of $1.2 \mathrm{~dB}$ by $\mathbf{S}_{4 b}$ and $10 \mathrm{~dB}$ by $\mathbf{S}_{8 b}$ at $B E R=10^{-5}$ ), compared to the respective OSTFCs of the same order, while they are superior to the conventional MB-OFDM. We note that this enhancement is gained without any increase of the total transmission power. Equivalently, the QOSTFCs may provide higher data rate with the same error performance as the OSTFCs. Second, $\mathbf{S}_{8 b}$ not only can provide higher system capacity (because of higher order as analyzed in Section I), compared to $\mathbf{S}_{4 b}$, but also can provide almost as good error performance as $\mathbf{S}_{4 b}$ in the channel models CM1 and $\mathrm{CM} 2$ at the same data rate. In the more dispersive channel models CM3 and CM4, there is a compromise between the system capacity and the code rate (as well as the error performance if we compare the two codes at the same data rate, because the decrease of the code rate of $\mathbf{S}_{8 b}$, compared to that of $\mathbf{S}_{4 b}$, causes the degradation of the error performance).

\section{CONCLUSIONS}

We have analyzed the application of QOSTFCs in our proposed STFC MB-OFDM UWB systems. Although only partial diversity can be achieved, QOSTFCs may still provide either a higher date rate or better error performance, compared to the full diversity OSTFCs of the same order. From the simulation results, it can be concluded that, for STFC MBOFDM UWB, QOSTFCs might be better than OSTFCs, with the penalty of higher decoding complexity, though both have relatively simple decoding complexity. Our future work would be the derivation of theoretical results to supplement the simulation results in this paper for complete examination of the advantages of the QOSTFC application in MB-OFDM UWB.

\section{ACKNOWLEDGMENT}

Dr. L. C. Tran would like to thank the Alexander von Humboldt (AvH) Foundation, Germany, for its support of this work.

\section{REFERENCES}

[1] T.-H. Tan and K.-C. Lin, "Performance of space-time block coded MBOFDM UWB systems," Proc. 4th Annual Communication Networks and Services Research Conference (CNSR'06), pp. 323 - 327, May 2006.

[2] S. M. Alamouti, "A simple transmit diversity technique for wireless communications," IEEE J. Select. Areas Commun., vol. 16, no. 8, pp. 1451 - 1458, Oct. 1998.

[3] W. P. Siriwongpairat, W. Su, M. Olfat, and K. J. R. Liu, "MultibandOFDM MIMO coding framework for UWB communication systems," IEEE Trans. Sign. Process., vol. 54, no. 1, pp. $214-224$, Jan. 2006.

[4] L. C. Tran and A. Mertins, "Space-time frequency code implementation in MB-OFDM UWB communications: design criteria and performance," accepted for publication in IEEE Trans. Wireless Commun. Available at http://www.isip.uni-luebeck.de/index.php?id=278, 2007.

[5] L. C. Tran, A. Mertins, E. Dutkiewicz, and X. Huang, "Space-timefrequency codes in MB-OFDM UWB communications: Advanced order8 STFC and its performance," Proc. 7th IEEE International Symposium on Communications and Information Technologies ISCIT 2007, Oct. 2007.

[6] V. Tarokh, N. Seshadri, and A. R. Calderbank, "Space-time codes for high data wireless communications: performance criterion and code construction," IEEE Trans. Inform. Theory, vol. 44, no. 2, pp. 744 765, Mar. 1998.

[7] H. Jafarkhani, "A quasi-orthogonal space-time block codes," IEEE Trans. Commun., vol. 49, no. 1, pp. $1-4$, Jan. 2001.

[8] S. Das, N. Al-Dhahir, and A. Calderbank, "Novel full-diversity highrate STBC for 2 and 4 transmit antennas," IEEE Commun. Lett., vol. vol. 10, pp. pp. 171-173, Mar. 2006.

[9] H. N. N. Duy, H. N. Ha, and H. D. Tuan, "High-rate space-time block coding schemes," Proc. IEEE 2nd International Conference on Communications and Electronics (ICCE2008), pp. 176-179, June 2008.

[10] L. C. Tran, T. A. Wysocki, A. Mertins, and J. Seberry, Complex Orthogonal Space-Time Processing in Wireless Communications, Springer, New York, USA, 2006.

[11] O. Tirkkonen and A. Hottinen, "Square-matrix embeddable space-time blocks codes for complex signal constellations," IEEE Trans. Inform. Theory, vol. 48, no. 2, pp. 384 - 395, Feb. 2002.

[12] WiMedia, "Multiband OFDM physical layer specification," WiMedia Alliance, Release 1.1, July 2005.

[13] L. C. Tran, A. Mertins, and T. A. Wysocki, "Quasi-orthogonal spacetime-frequency codes in MB-OFDM UWB communications," Proc. International Conference on Signal Processing and Communication Systems ICSPCS2007, Dec. 2007.

[14] L. C. Tran and A. Mertins, "On the use of quasi-orthogonal space-timefrequency codes in MB-OFDM UWB," Proc. 2nd IEEE International Conference on Communication and Electronics (ICCE2008), June 2008.

[15] J. Foerster et. al., "Channel modelling sub-committee report final," IEEE P802.15 Working Group for Wireless Personal Area Networks (WPANs), IEEE P802.15-02/490r1-SG3a, Oct. 2005. 